\title{
Oxygen permeability of the pigmented material used in cosmetic daily disposable contact lenses
}

\author{
This article was published in the following Dove Press journal: \\ Clinical Ophthalmology \\ 8 December 2016 \\ Number of times this article has been viewed
}

\section{Stephen Galas \\ Lenora L Copper}

Johnson \& Johnson Vision Care Inc., Jacksonville, FL, USA
Correspondence: Lenora L Copper Johnson \& Johnson Vision Care Inc., 7500 Centurion Parkway, Suite 100, Jacksonville, FL32256, USA Email lcopper2@its.jnj.com
Purpose: To evaluate the individual contributions of pigment colorant and packing solution containing polyvinyl pyrrolidone (PVP) on the oxygen permeability (Dk) of a cosmetic printed etafilcon A daily disposable contact lens packaged with PVP.

Method: The oxygen transport of a contact lens is evaluated through the central optical zone of the lens. Cosmetic printed contact lenses contain pigment colorant in the periphery or midperiphery of the lens. Therefore, to assess the impact of cosmetic print on oxygen permeability, special lenses need to be produced that contain the colorant within the central optical zone. This technique was used to obtain multiple measurements of nonedge-corrected $\mathrm{Dk} / \mathrm{t}$ of both the center pigmented lens and its nonpigmented equivalent, using a polarographic measurement described in International Organization for Standardization (ISO) 18369-4:2006(E), and the Dk derived for each measurement is corrected for edge effect. In addition, the edge-corrected Dk values of lenses made from the same monomer batch were measured. The lenses were packaged and autoclaved with and without proprietary technology which embeds PVP in the contact lens during autoclaving. The resulting Dk value of the printed lens material was then used with thickness data to generate true $\mathrm{Dk} / \mathrm{t}$ profiles for a given lens power.

Results: The edge-corrected Dk of the printed etafilcon A lens with offset pigment colorant was measured to be $19.7 \times 10^{-11}\left(\mathrm{~cm}^{2} / \mathrm{s}\right)\left(\mathrm{mL} \mathrm{O}_{2} / \mathrm{mL} \cdot \mathrm{mmHg}\right)$ at $35^{\circ} \mathrm{C}$. This was within $\pm 20 \%$ tolerance range as specified in ISO 18369-2:2012(E) for the edge-corrected Dk of the nonpigmented etafilcon A control lens evaluated during the same session, $19.5 \times 10^{-11}\left(\mathrm{~cm}^{2} / \mathrm{s}\right)\left(\mathrm{mL} \mathrm{O}_{2} / \mathrm{mL} \cdot \mathrm{mmHg}\right)$. The edge-corrected Dk values of the lenses packaged with PVP (mean 20.1, standard deviation [SD] 0.3) were also within the $\pm 20 \%$ tolerance range compared to those packaged without PVP (mean 20.0, SD 0.3).

Conclusion: The pigment colorant and PVP embedded in the contact lens during autoclaving were not found to influence the oxygen permeability of the etafilcon A material.

Keywords: oxygen, oxygen permeability, Dk, hydrogel contact lenses, cosmetic contact lenses

\section{Introduction}

In the absence of a contact lens, the bulk of the oxygen required to maintain normal corneal metabolism is derived directly from the atmosphere. However, the introduction of a contact lens onto the eye forms a barrier, potentially reducing oxygen transport to the cornea. Oxygen deprivation may result in hypoxic events such as corneal swelling, ${ }^{1}$ limbal hyperemia, ${ }^{2}$ corneal vascularization, ${ }^{3,4}$ and epithelial thinning. ${ }^{5}$ Consequently, suitable measures of oxygen transfer are important in the development and subsequent prescribing of contact lenses.

The most widely used measures of oxygen transfer are those of oxygen permeability and oxygen transmissibility. Oxygen permeability (Dk) is a property of a contact lens material and is independent of lens geometry. This measure is derived from the product of the diffusion coefficient (D) of the material and the oxygen solubility (k). 
Oxygen transmissibility (Dk/t), by contrast, relates to a specific lens design and can be determined by dividing the $\mathrm{Dk}$ value of the lens material by a local or averaged thickness $(t)$ of the lens. $\mathrm{Dk} / \mathrm{t}$ is a more clinically relevant measure than Dk since it allows for a direct comparison of oxygen transport between specific lens types. Manufacturers typically quote a single figure for the $\mathrm{Dk} / \mathrm{t}$ value based on the center thickness of a $-3.00 \mathrm{D}$ version of a given lens. However, while providing some relative comparison between lens types, this value is not representative of differences seen across different lens power profiles and different geometries. Alternative, and arguably more clinically relevant, measures of oxygen transfer such as oxygen flux, ${ }^{6}$ equivalent oxygen potential, ${ }^{7}$ and oxygen consumption, ${ }^{8}$ have also been quoted by researchers; however, $\mathrm{Dk}$ and $\mathrm{Dk} / \mathrm{t}$ remain widespread metrics in terms of quantifying oxygen transport through contact lenses.

Although several different methods have been described in the literature for the measurement of Dk and Dk/t, the most commonly used for hydrogel contact lens materials is the polarographic method, ${ }^{9}$ as defined in the American National Standards Institute (ANSI) and the International Organization for Standardization (ISO) standards. ${ }^{10,11} \mathrm{The} \mathrm{Dk} / \mathrm{t}$ for a contact lens of known thickness is first determined. The $\mathrm{Dk} / \mathrm{t}$ of additional samples with significantly different thickness values for a stack of two or more samples ${ }^{12}$ are then measured, and then converted into a Dk value taking into account the mean thickness in the area in which the stack is measured. In the polarographic or the so-called "Fatt method", the contact lens sample is held against a Clark-type electrode and the front surface of the lens sample is exposed to a water saturated air environment to prevent lens dehydration. Measurements are undertaken in controlled temperature and humidity conditions. However, the measurement of Dk is complicated by a number of potential sources of error, particularly edge and boundary effects..$^{13}$

The edge effect occurs as a result of the lateral diffusion of oxygen from the larger surface area of the anterior lens into the smaller cathode sensor area posteriorly, resulting in an overestimation of the Dk value. The edge effect can be compensated by the application of correction factors. ${ }^{14}$ The boundary effect, by contrast, occurs when a thin film of saline lines the anterior and/or posterior surface of the lens sample, thus creating an additional layer of resistance for oxygen diffusion, resulting in an underestimation of Dk. The boundary effect can be corrected by the measurement of multiple stacked samples of the same material, but in differing thicknesses. The error due to the boundary effect can be determined (and subsequently compensated for) by plotting the reciprocal transmissibilities against the lens thickness, as described in ANSI and ISO documents. ${ }^{10,11}$ In addition, contact lenses with a uniform thickness profile in the area of measurement can be stacked on top of one another to create the different thickness values. The Dk values of the hydrogel material calculated from the stacked lenses were equivalent to those of the hydrogel material calculated using monolithic lenses of the same material. ${ }^{12}$

The addition of a colorant, such as that used in enclosed or dot-matrix printed contact lens designs, might be expected to act like a barrier and therefore reduce oxygen transmissibility for a given lens. The oxygen permeability of a soft contact lens is typically measured in the central portion of the sample because of its uniform thickness. However, the printed portion of a cosmetic contact lens is typically located in the periphery (or mid-periphery) of the lens, a region which does not allow for an accurate measurement of Dk due to the wide variations in thickness values across a given lens profile (Figure 1).

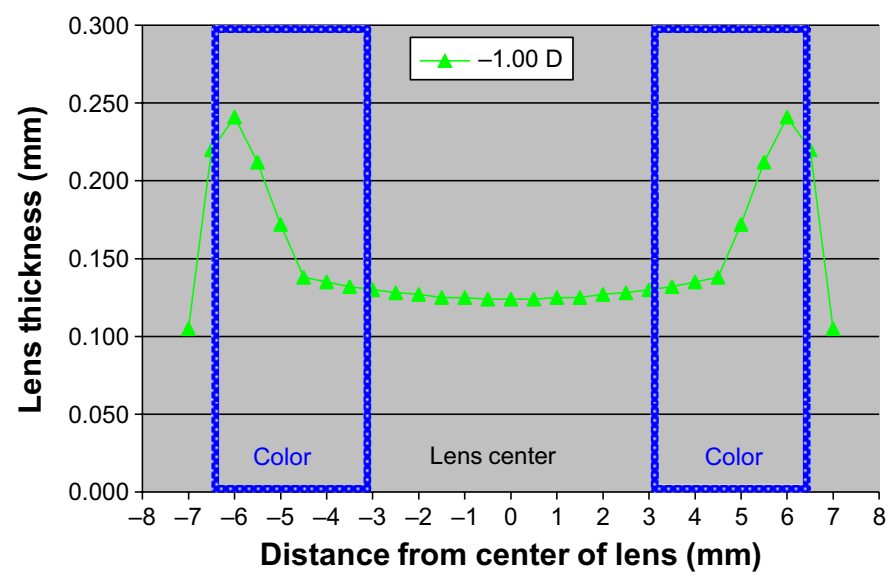

Figure I Thickness profile of a currently marketed -I.00 D cosmetic print contact lens (I-Day Acuvue ${ }^{\circledR}$ Define $^{\circledR}$ ). Note: Blue boxes indicate the positioning of the colorant. 
Previous workers ${ }^{15}$ have attempted to address this difficulty by taking measurements through both the clear and pigmented portions of the lens and averaging the results. However, this approach does not provide an accurate measurement of $\mathrm{Dk}$ due to the large thickness gradient in the pigmented region of the lens and, more importantly, it is not a direct measurement of the region of interest (central, optical zone).

The oxygen permeability of etafilcon A contact lens matrices is essentially controlled by the water content of the contact lens polymer itself. Polyvinyl pryrrolidone (PVP) is a hydrophilic polymer capable of increasing the water content of etafilcon A lenses if present in the contact lens matrix in significant amounts. The PVP, used as a packing solution additive by Johnson and Johnson Vision Care, Inc., is present in extremely low concentrations in solution, and an even smaller amount is actually embedded by the contact lens (data on file Johnson and Johnson Vision Care, 2013). Given that PVP itself does not have a high affinity for oxygen and that the low levels present in contact lens matrices cannot significantly alter the lens water contents, it is unlikely that Dk of etafilcon A lenses with PVP embedded in the contact lens during autoclaving will impact $\mathrm{Dk} / \mathrm{t}$ values of the product, but confirmation of this by laboratory testing would be desirable.

The purpose of this study was to evaluate the individual contributions of pigment colorant material and the PVP embedded in the contact lens during autoclaving on the oxygen permeability (Dk) of a cosmetic printed etafilcon A daily disposable contact lens packaged with PVP.

\section{Method}

Specially modified test lenses were manufactured with the pigmented component of the lens offset and relocated at the center of the lens. The pigmented component was enclosed within the lens matrix, and not present on the lens surface, as is the case with the commercially available 1-Day Acuvue ${ }^{\circledR}$ Define $^{\circledR}$ brand contact lenses (Johnson \& Johnson Vision Care Inc., Jacksonville, FL, USA) (cosmetically printed etafilcon A). ${ }^{16}$

The preferred lens geometry used in the measurement of Dk is a uniform thickness profile across the central portion of the lens. Lenses of low power meet this requirement so consequently, -0.50 and $-1.00 \mathrm{D}$ lenses (Table 1) were used throughout this investigation. Lenses which are uniformly thick across the measurement area also eliminate the alignment error associated with ensuring that the center of the lens is positioned over the center of the sensor.

Measurements were made using the polarographic (Fatt) method described in ISO 18369-4:2006(E). ${ }^{11}$ Lenses of predetermined thickness were equilibrated at $35^{\circ} \mathrm{C}$, and placed directly onto the polarographic sensor to achieve electrical equilibrium (Figure 2).

Once electrically equilibrated, multiple measurements of nonedge-corrected $\mathrm{Dk} / \mathrm{t}$ were made for both the test lenses and the control lenses. Lenses were added to the stack one at a time and further measurements were taken sequentially (with a mimimum of four), such that the effect of thickness on $\mathrm{Dk} / \mathrm{t}$ could be established and corrected for the boundary effect. The resulting measurements were also edge-corrected in the calculation to provide a true estimate of the Dk value. Evaluations of both the test and the control lenses were undertaken on the same day so as to minimize the effect of any equipment or environmental changes. All measurements were undertaken by a single operator. The procedure was consistent for all lenses in the study.

The resulting Dk value of the pigmented lens material was subsequently used with proprietary lens thickness values to generate true $\mathrm{Dk} / \mathrm{t}$ profiles for different lens powers for the cosmetic printed etafilcon A lens.

Experiment 1 evaluated the individual contributions of pigment colorant material on the oxygen permeability ( $\mathrm{Dk}$ ) by comparing the modified cosmetically printed etafilcon $\mathrm{A}$ "Vivid" (Test 1) to 1-Day Acuvue ${ }^{\circledR}$ Moist (etafilcon A with PVP) (Control 1). Experiment 2 evaluated the individual contributions of the PVP embedded in the contact lens during autoclaving on the oxygen permeability (Dk) by comparing nonprinted lenses with PVP (Test 2) to nonprinted lenses with no PVP (Control 2) (Table 1).

\section{Results}

The edge-corrected oxygen permeability of the cosmetically printed etafilcon A with pigmented offset in the lens

Table I Study lenses

\begin{tabular}{|c|c|c|c|c|}
\hline Lens & $\begin{array}{l}\text { Material/water } \\
\text { content }\end{array}$ & $\begin{array}{l}\text { Base curvel } \\
\text { diameter }\end{array}$ & Lens power (D) & $\begin{array}{l}\text { Average center } \\
\text { thickness }(\mathrm{mm})\end{array}$ \\
\hline Modified I-Day Acuvue ${ }^{\circledR}$ Define ${ }^{\circledR}$ “Vivid” (Test I) & Etafilcon A (58\%) & $8.50 / 14.20$ & -0.50 & 0.128 \\
\hline I-Day Acuvue ${ }^{\circledR}$ Moist (Control I) & Etafilcon A (58\%) & $8.50 / 14.20$ & -0.50 & 0.126 \\
\hline Nonprinted lens with PVP (Test 2) & Etafilcon A (58\%) & $8.30 / 14.00$ & -0.50 & 0.113 \\
\hline Nonprinted lens with no PVP (Control 2) & Etafilcon A (58\%) & $8.30 / 14.00$ & -0.50 & 0.113 \\
\hline
\end{tabular}

Abbreviation: PVP, polyvinyl pyrrolidone. 


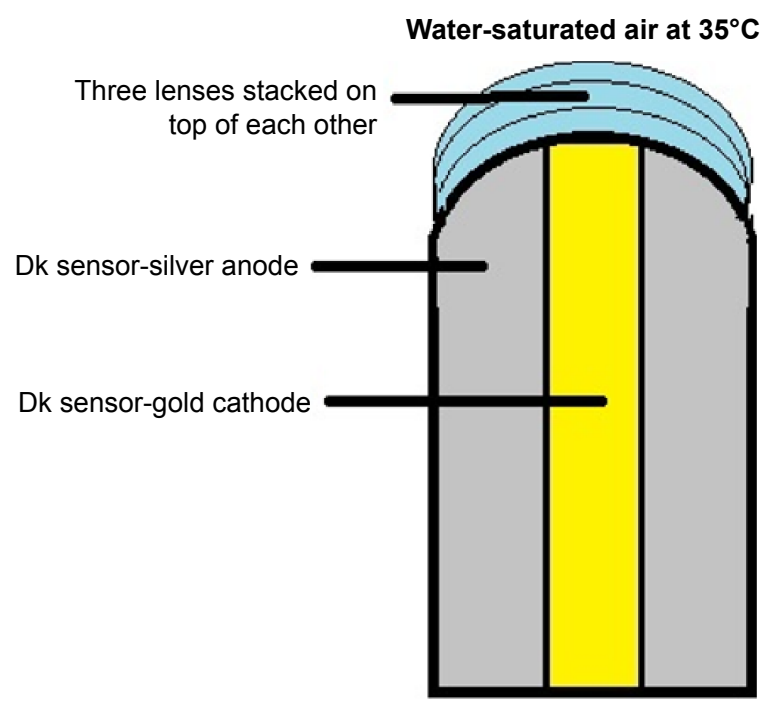

Figure 2 Lens stack mounted on the polarographic sensor for measurement.

center (Test 1) was measured to be $19.7 \times 10^{-11}\left(\mathrm{~cm}^{2} / \mathrm{s}\right)$ $(\mathrm{mL} \mathrm{O} / \mathrm{mL} \cdot \mathrm{mmHg})$ at $35^{\circ} \mathrm{C}$. This was within $\pm 20 \%$ of the nominal reported value for 1-Day Acuvue ${ }^{\circledR}$ (etafilcon A without PVP), 21.4 $\pm 4.310^{-11}\left(\mathrm{~cm}^{2} / \mathrm{s}\right)\left(\mathrm{mL} \mathrm{O}_{2} / \mathrm{mL} \cdot \mathrm{mmHg}\right)$ and 1-etafilcon A with PVP (Control 1) in this experiment $\left(19.5 \times 10^{-11}\left(\mathrm{~cm}^{2} / \mathrm{s}\right)\right.$ $\left(\mathrm{mL} \mathrm{O}_{2} / \mathrm{mL} \cdot \mathrm{mmHg}\right)$. The oxygen permeability values of the pigmented study lens (Test 1) and the nonpigmented control lens (Control 1) are summarized in Table 2.

The edge-corrected oxygen permeability of the etafilcon A lenses with PVP (Test 2) was measured to be $20.1 \times 10^{-11}$ $\left(\mathrm{cm}^{2} / \mathrm{s}\right)\left(\mathrm{mL} \mathrm{O}_{2} / \mathrm{mL} \cdot \mathrm{mmHg}\right)$ at $35^{\circ} \mathrm{C}$. This was within $\pm 20 \%$ of the nominal reported value for etafilcon A without PVP, $21.4 \pm 4.310^{-11}\left(\mathrm{~cm}^{2} / \mathrm{s}\right)\left(\mathrm{mL} \mathrm{O}_{2} / \mathrm{mL} \cdot \mathrm{mmHg}\right)$ and similar to the value of the control lens made from the same monomer batch and processed without PVP (Control 2$)\left(20.0 \times 10^{-11}\left[\mathrm{~cm}^{2} / \mathrm{s}\right]\right.$ $\left[\mathrm{mL} \mathrm{O}_{2} / \mathrm{mL} \cdot \mathrm{mmHg}\right]$ at $\left.35^{\circ} \mathrm{C}\right)$.

The oxygen transmissibility (Dk/t) of a lens was calculated from the measured edge-corrected oxygen permeability (Dk) of the material divided by the center thickness of the lens sample of interest.

The nominal edge-corrected Dk of an etafilcon A material is $21.4 \times 10^{-11}\left(\mathrm{~cm}^{2} / \mathrm{s}\right)\left(\mathrm{mL} \mathrm{O}_{2} / \mathrm{mL} \cdot \mathrm{mmHg}\right)$. The $\mathrm{Dk} / \mathrm{t}$ of the $-3.00 \mathrm{D}$ lens at the center was calculated by dividing the Dk of the material $\left(21.4 \times 10^{-11}\left[\mathrm{~cm}^{2} / \mathrm{s}\right]\left[\mathrm{mL} \mathrm{O}_{2} / \mathrm{mL} \cdot \mathrm{mmHg}\right]\right)$ by the center thickness of the lens $(0.0084 \mathrm{~cm})$. The Dk/t is $25 \times 10^{-9}(\mathrm{~cm} / \mathrm{s})\left(\mathrm{mL} \mathrm{O}_{2} / \mathrm{mL} \cdot \mathrm{mmHg}\right)$. Colloquially, this lens is said to have a Dk/t of 25 .

The thickness profile of a currently marketed $-1.00 \mathrm{D}$ printed cosmetic lens (1-cosmetically printed etafilcon $\mathrm{A}$ ) that is shown in Figure 1 was used to construct an oxygen transmissibility profile for the same lens, using the nominal Dk value. The oxygen transmissibility profile is shown in Figure 3.

\section{Discussion}

The pigmented portion of a cosmetic printed contact lens is typically located in the periphery or mid-periphery of the lens, which does not allow for evaluation of the effect of print on the oxygen permeability. A technique was developed to overcome this difficulty, whereby specially modified lenses were manufactured with the pigment colorant offset from the normal location and relocated at the center of the lens to facilitate measurement of the material containing the pigment. This study sought to more accurately evaluate the oxygen permeability of a cosmetic printed lens, with PVP, in comparison with that of its nonpigmented equivalent. The edge-corrected Dk value for the cosmetic printed etafilcon A lens in this study was within $\pm 20 \%$ of the Dk value for the nonpigmented control, as per the measurement tolerance level set by ANSI and ISO standards. ${ }^{10,11}$ As such, there was no difference in Dk between the pigmented and nonpigmented study lenses. However, the fact that there was no difference in oxygen permeability between the enclosed pigmented etafilcon A lens and its nonpigmented equivalent assessed in this study does not mean that this effect would necessarily be true for all cosmetic contact lenses. The manufacturing processes, print/tinting composition, and print thicknesses (pigment load) employed in other enclosed, or even dot-matrix, design cosmetic lenses may still impact oxygen permeability with clinical implications for the wearer.

The fact that there was no effect on Dk due to the addition of the pigment in this study was, perhaps, surprising, especially given that previous workers ${ }^{15}$ found a significant

Table 2 Oxygen permeability (Dk) results

\begin{tabular}{|c|c|c|c|}
\hline \multirow[t]{2}{*}{ Lens brand } & \multicolumn{2}{|c|}{$\begin{array}{l}\text { Oxygen permeability (Dk) } \\
\left.\text { (value }\left[\mathrm{cm}^{2} / \mathrm{s}\right]\left[\mathrm{mL} \mathrm{O}_{2} / \mathrm{mL} \cdot \mathrm{mmHg}\right] \text { at } 35^{\circ} \mathrm{C}\right)\end{array}$} & \multirow[t]{2}{*}{ Results } \\
\hline & Edge-corrected & Nonedge-corrected* & \\
\hline Nominal value for I-Day Acuvue ${ }^{\circledR}$ & $(21.4 \pm 4.3) \times 10^{-11}$ & $(28.0 \pm 5.6) \times 10^{-11}$ & Reported value \\
\hline Modified I-Day Acuvue ${ }^{\circledR}$ Define ${ }^{\circledR}$ "Vivid" style (Test I) & $19.7 \times 10^{-11}$ & $27.3 \times 10^{-11}$ & No difference \\
\hline I-Day Acuvue ${ }^{\circledR}$ Moist $^{\circledR}($ Control I) & $19.5 \times 10^{-11}$ & $26.9 \times 10^{-11}$ & No difference \\
\hline
\end{tabular}

Note: $*$ The $20 \%$ tolerance for the nonedge-corrected value is for comparison purpose only. 


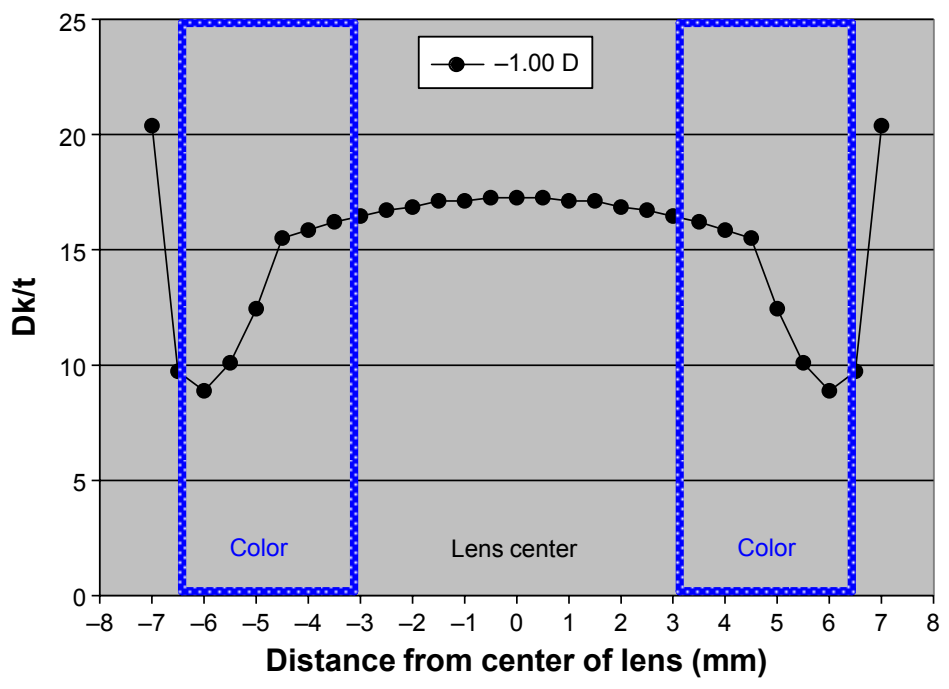

Figure 3 Calculated Dk/t profile of a -I.00D I-Day Acuvue ${ }^{\circledR}$ Define ${ }^{\circledR}$.

Notes: The Dk/t units are $10^{-9}(\mathrm{~cm} / \mathrm{s})\left(\mathrm{mL} \mathrm{O}_{2} \times \mathrm{mmHg}\right)$. The nominal value of Dk was used in the calculation.

Abbreviations: $\mathrm{Dk}$, oxygen permeability; $t$, thickness of the lens.

increase in corneal edema for wearers of a laminated cosmetic tinted lens. However, it is likely that improved manufacturing processes, making the etafilcon A cosmetic printed contact lens thickness profile similar to that of the clear etafilcon A lens, may have facilitated this.

The Dk of a hydrogel material like etafilcon A is typically correlated with the water content of the material. ${ }^{17} \mathrm{An}$ increase in water content would correlate to an increase in $\mathrm{Dk}$ value. Conversely, a decrease in water content would result in a lower Dk value. Although the addition of some types of components to a blister packing solution may influence water content of a lens material, the PVP embedded in the contact lens during autoclaving had no such effect on the etafilcon A material and consequently did not change the Dk of the etafilcon A contact lenses.

Oxygen transmissibility $(\mathrm{Dk} / \mathrm{t})$ is a property of a specific lens and is calculated from the measured $\mathrm{Dk}$ value of the lens material and the thickness of the lens. The minimum $\mathrm{Dk} / \mathrm{t}$ values in this experiment occurred where the lens had a maximum thickness, toward the edge of the lens and in the region where the pigment is located. The smaller $\mathrm{Dk} / \mathrm{t}$ values in the printed regions shown in Figure 3 are due to the thickness increase in the same region, as shown by Figure 1, and not by a change in the Dk of the material.

\section{Conclusion}

In conclusion, this study has shown that the pigment colorant and the PVP embedded in the contact lens during autoclaving were not found to influence the oxygen permeability of the etafilcon A material.

\section{Acknowledgments}

We would like to thank Lee Anthony Hall, MCOptom PhD, who served as the lead medical writer for this manuscript. Additionally, we would like to thank Shivkumar Mahadevan $\mathrm{PhD}$, Kathy Osborn Lorenz OD MS, Meredith E Jansen OD MS, and Michelle Mundorf PhD for their valuable input in drafting this manuscript; and Ruon Phlong for sample analysis. Finally, we would like to acknowledge Karin McCarthy, FAAO, for her support in this work.

\section{Disclosure}

Both authors were employed by Johnson \& Johnson Vision Care at the time of data collection. L Copper continues to be employed by Johnson \& Johnson.

\section{References}

1. Erickson P, Comstock TL, Zantos SG. Effects of hydrogel lens transmissibility profiles on local corneal swelling during eye closure. Optom Vis Sci. 1996;73:169-177.

2. Papas EB. The role of hypoxia in the limbal vascular response to soft contact lens wear. Eye Cont Lens. 2003;29:S72-S74; discussion S83-S84, S192-S194.

3. Holden BA, Sweeney DF, Vannas A, Nilsson KT, Efron N. Effects of long-term extended contact lens wear on the human cornea. Invest Ophthalmol Vis Sci. 1985;26:1489-1501.

4. Holden BA, Sweeney DF, Swarbrick HA, et al. The vascular response to long-term extended contact lens wear. Clin Exp Optom. 1986;69: 112-119.

5. Perez JG, Meijome JM, Jalbert I, Sweeney DF, Erickson P. Corneal epithelial thinning profile induced by long-term wear of hydrogel lenses. Cornea. 2003;22:304-307.

6. Fatt I. Measurement of oxygen flux into the cornea by pressing a sensor onto a soft contact lens on the eye. Am J Optom Physiol Opt. 1978;55: 294-301.

7. Hill R. The cornea's need to breathe. Int Cont Clin. 1976;3:60-64. 
8. Brennan NA. Total corneal oxygen consumption as an index of corneal oxygenation during contact lens wear. Optom Vis Sci. 2005;82: $467-472$.

9. Fatt I, St Helen R. Oxygen tension under an oxygen-permeable contact lens. Am J Optom Arch Am Acad Optom. 1971;48:545-555.

10. ANSI Standard Z80.20-2010. Contact lenses - Standard terminology, tolerances, measurements and physicochemical properties. 2010.

11. ISO Standard 18369-4:2006. Contact Lenses - Part 4: Physicochemical properties of contact lens materials. 2006.

12. Weissman BA, Fatt I. Stacking samples while measuring oxygen transmissibility of hydrogel contact lenses. Optom Vis Sci. 1989;66: 235-238.

13. Morgan PB, Efron N. The oxygen performance of contemporary hydrogel contact lenses. Cont Lens Ant Eye. 1998;21:3-6.
14. Fatt I, Rasson JE, Melpolder JB. Measuring oxygen permeability of gas permeable hard and hydrogel lenses and flat samples in air. Int Contact Lens Clin. 1987;14:389-402.

15. Gauthier CA, Grant T, Holden BA. Clinical performance of two opaque, tinted soft contact lenses. J Am Optom Assoc. 1992;63:344-349.

16. Lorenz KO, Kakkassery J, Boree D, Pinto D. Atomic force microscopy and scanning electron microscopy analysis of daily disposable limbal ring contact lenses. Clin Exp Optom. 2014;97:411-417.

17. Young MD, Benjamin WJ. Calibrated oxygen permeability of 35 conventional hydrogel materials and correlation with water content. Eye Cont Lens. 2003;29:126-133.
Clinical Ophthalmology

\section{Publish your work in this journal}

Clinical Ophthalmology is an international, peer-reviewed journal covering all subspecialties within ophthalmology. Key topics include: Optometry; Visual science; Pharmacology and drug therapy in eye diseases; Basic Sciences; Primary and Secondary eye care; Patient Safety and Quality of Care Improvements. This journal is indexed on

\footnotetext{
Submit your manuscript here: http://www.dovepress.com/clinical-ophthalmology-journal
}

\section{Dovepress}

PubMed Central and CAS, and is the official journal of The Society of Clinical Ophthalmology (SCO). The manuscript management system is completely online and includes a very quick and fair peer-review system, which is all easy to use. Visit http://www.dovepress.com/ testimonials.php to read real quotes from published authors. 